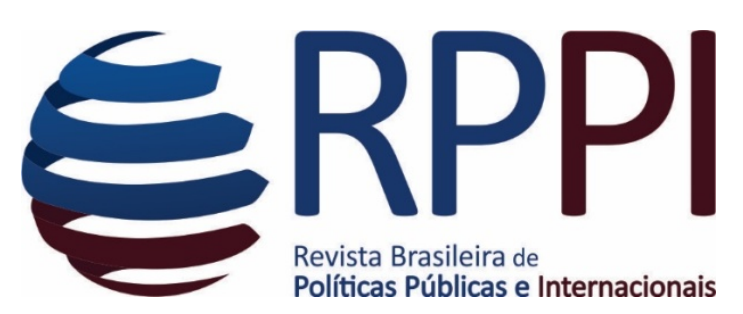

Data de recebimento: 16/02/2018

Data de aceite: 23/06/2018

Organização: Programa de Pós-Graduação em

Gestão Pública e Cooperação Internacional

(PGPCI/UFPB)

Avaliação: Por Pares

\title{
O Balanced Scorecard como Ferramenta Estratégica para Pequenos Municípios
}

\section{The Balanced Scorecard as Strategic Tool for Small Municipalities}

\author{
Marinês Taffarel \\ Departamento de Ciências Contábeis (DECIC/I) - Universidade Estadual do Centro-Oeste \\ (UNICENTRO) \\ E-mail: marinestaffarel@gmail.com / marinestaffarel@yahoo.com.br
}

\begin{abstract}
Resumo: A Administração Pública requer de forma sistemática mecanismos de eficiência e eficácia na execução de suas ações. A Lei de Responsabilidade Fiscal proporcionou um completo e profundo reordenamento das finanças públicas, entretanto não apresenta uma visão de longo prazo, especialmente no que diz respeito à conformação estratégica dos órgãos públicos, orientada para elevação consistente de seu desempenho. O objetivo geral desta pesquisa consiste em apresentar uma estrutura de implantação do Balanced Scorecard e analisar as vantagens e benefícios, bem como os principais obstáculos à sua implantação nos pequenos municípios. Os procedimentos metodológicos são de pesquisa descritiva, bibliográfica e documental. Aestrutura do Balanced Scorecard proposta é construída a partirdo modelo aplicado no setor privado por Kaplan e Norton e adaptado às particularidades legais e necessidades gerenciais da gestão pública municipal. Os resultados da pesquisa indicam que a implantação do Balanced Scorecard como instrumento de tradução da visão, planos e estratégia de governo pode proporcionar inúmeros benefícios, ao contemplar medidas de efetividade, eficiência, eficácia e economicidade dos serviços públicos.
\end{abstract}

Palavras-chave: Balanced Scorecard,Administração pública,Pequenos municípios.

\begin{abstract}
The Public Administration requires a systematic mechanism for efficiency and effectiveness in carrying out actions. The Lei de Responsabilidade Fiscal has provided a complete and thorough reorganization of public finance, however it does not present a long-term view, especially in regard to the strategic shaping of public bodies, oriented consistently to higherperformance. The objective of this research is to provide a framework for implementation of the Balanced Scorecardand to analyze the advantages and benefits as well as the main obstacles forits implementation in small city governments. The methodological procedures are descriptive, literature reviewing and documental. The proposed structure of the Balanced Scorecardis built from the model applied in the private sector by Kaplan and Norton and adapted to the particular legal and managerial needs of municipal public administration. The survey results indicate that the implementation of the Balanced Scorecard as a translation tool of the
\end{abstract}


TAFFAREL, Marinês. O Balanced Scorecard como Ferramenta Estratégica para Pequenos Municípios.

vision, strategy and plans of government can provide numerous benefits to the extent that focuses on the measures of efficiency, effectiveness and economical use of public resources.

Keywords: Balanced Scorecard, Public administration, Small sized municipalities.

\section{Introdução}

A administração pública brasileira é regida pelos princípios constitucionais de legalidade, impessoalidade, moralidade, publicidade e eficiência. Apesar de as organizações do setor público não se orientarem pela lucratividade ou retorno do capital, é exigido destas, resultados otimizados na aplicação dos recursos, adotando-se, para tanto, padrões de eficiência e eficácia comparáveis aos aplicados no setor privado. Estes padrões estão em conformidade com a teoria da New Public Management (NPM) ou Nova Administração Pública que surgiu na década de 80. O movimento do NPM, de acordo com Pinto (2008), procura dar ênfase no incremento da qualidade e na cobertura dos serviços públicos como meta central, em que o cidadão passou a ser o cliente foco da gestão pública.

No Brasil, a gestão dos recursos públicos, especialmente a partir da Lei de Responsabilidade Fiscal (LRF), dezembro de 2000, passou a requerer maior necessidade de controles e sistemas de informação coordenados com os objetivos públicos. Para Matias-Pereira (2006), a LRF objetiva melhorar a qualidade das ações de gestão dos recursos públicos. A partir de sua implantação, além da necessidade de aplicação de controles gerenciais, os gestores públicos tornaram-se obrigados a conciliar os princípios constitucionais que norteiam as atividades públicas com os preceitos da gestão fiscal responsável.

A LRF preconiza que o orçamento público anual, instrumento de execução das metas e objetivos previstos para determinado exercício, deve ser compatível com os anexos de metas fiscais e de riscos fiscais previstos pela LDO e, também, ter compatibilidade com o PPA vigente. Santana et al. (2007) refere que a LRF estabelece mecanismos norteadores para fomentar grandes avanços na política de planejamento brasileira, inserindo no seu texto o elo entre orçamento, a

Revista Brasileira de Políticas Públicas e Internacionais, v.3, n.2, Dezembro/2018, pp. 59-80. 
TAFFAREL, Marinês. O Balanced Scorecard como Ferramenta Estratégica para Pequenos Municípios.

programação financeira e o fluxo de caixa, além de instrumento de acompanhamento, avaliação e ajuste do que foi planejado e orçado. No entanto, os municípios de pequeno porte, de modo geral, parecem estar distantes deum planejamento eficiente, eficaz e efetivo, capaz de incorporar as novas práticas. São identificadas inúmeras dificuldades, até mesmo, para elaborar de forma adequada o orçamento, uma das principais ferramentas de planejamento e de controle utilizadas pela administração pública.

A LRF reforçou a necessidade de implantação de um sistema de controle interno, tal como preconizado na Constituição Federal de 1988 e pela Lei n. 4.320(1964). O objetivo do controle interno é atuar como um instrumento de auxílio para o administrador público e mecanismo de proteção e defesa dos interesses do cidadão. Dentre as principais funções do sistema de controle interno encontra-se a atribuição de assegurar a eficiência, efetividade, eficácia e economicidade dos serviços públicos, acompanhar os limites de gastos, endividamento e a execução orçamentária, financeira e patrimonial dos municípios. No entanto, o sistema de controle interno, principalmente nos pequenos municípios, ainda encontra dificuldade de implantação e funcionamento efetivo.

Para Bergue (2005) a Administração Pública necessita de diretrizes de gestão orientadas para a adição de valor ao produto da ação estatal, por meio do incremento qualitativo e quantitativo na produção de riqueza pública e geração de bens e serviços públicos de qualidade. Neste sentido, não é devido ao pequeno porte populacional de um município ou à reduzida dimensão de sua estrutura administrativa que as políticas de gestão se tornam desnecessárias. Nestes municípios, possivelmente, mais relevantes sem os trem as ações governamentais pautadas pela ação planejada e controlada.

Por seu turno, o Balanced Scorecard (BSC) é um sistema consagrado de gestão estratégica que interliga a estratégia organizacional com os objetivos estabelecidos (Kaplan \& Norton, 1997). Isso significa que o BSC é um sistema orientado para moldar estrategicamente a estrutura das organizações no sentido de promover o alcance dos objetivos e metas de forma mais plena e mais econômica. Em resumo, o BSC é uma valiosa ferramenta para melhorar o

Revista Brasileira de Políticas Públicas e Internacionais, v.3, n.2, Dezembro/2018, pp. 59-80. 
TAFFAREL, Marinês. O Balanced Scorecard como Ferramenta Estratégica para Pequenos Municípios.

desempenho organizacional. Apesar disso, o BSC é pouco utilizado na Administração Pública e praticamente desconhecido nos pequenos municípios.

Na atualidade, o BSC é aplicado principalmente em empresas privadas, devido aos custos elevados e a falta de especialistas, que representam um obstáculo real na implantação deste instrumento em instituições públicas (Gabriel, Cheonea \& Mihaela,2017). Para Wen-Cheng (2013) as pesquisas raramente discutem os conceitos de Balanced Scorecard e a importância da análise desempenho para a melhoria do governo local. Assim, o Balanced Scorecard pode ser o mais adequado entre todas as ferramentas de gestão para o setor público (Gabriel,_Victoria \& Mihaela,_2017).

OBalanced Scorecard é destacadamente importante como direcionador estratégico para o setor público, pois é uma ferramenta de medição de desempenho, capaz de perceber as distintas dimensões da atividade do Estado, capturando as diferenças entre as atividades do setor público e do setor privado (Blonski, Prates\& Costa, 2017).

Estudos recentes (Dreveton, 2013), (Diel \& Bampi, 2016), (Annapurna \& Manchala, 2017), (Bobe, Mihret \& Obo, 2017), (Blonski, Prates\& Costa, 2017), (Monteiro\& Ribeiro, 2017) buscam evidenciar a importância do Balanço Scorecard como ferramenta de gestão na Administração Pública. O estudo de Dreveton (2013) buscou apresentar os benefícios do Balanced Scorecard para o controle e gerenciamento de uma organização do setor público na França. Diel e Bampi (2016) apresentaram uma proposta para a concepção simplificada de uma ferramenta capaz de gerar elementos subsidiários para a tomada de decisão em gestão pública, por meio da combinação de Benchmarking com o Balanced Scorecard.

O estudo de Annapurna e Manchala (2017) avaliou o desempenho dos três principais bancos do setor público na Índia, por meio da aplicação do Balanced Scorecard. Bobe, Mihret e Obo (2017) examinaram a adoção do BSC em uma grande organização do setor de saúde pública na Etiópia, como parte de um programa de implantação de um sistema unificado de planejamento estratégico e monitoramento de desempenho de todo o setor.

Blonski, Prates e Costa, (2017) por meio de um estudo de caso sobre a Receita Federal do Brasil, buscaram compreender como ocorre a gestão estratégica no governo federal brasileiro,

Revista Brasileira de Políticas Públicas e Internacionais, v.3, n.2, Dezembro/2018, pp. 59-80. 
TAFFAREL, Marinês. O Balanced Scorecard como Ferramenta Estratégica para Pequenos Municípios.

por meio da correspondência entre os preceitos da Nova Gestão Pública e o Balanceado Scorecard. Monteiro e Ribeiro (2017) buscaram apresentar as diferentes possibilidades expostas na literatura para a integração das questões ambientais no BSC, não apenas no setor privado, mas também no setor público, enfatizando a necessidade de adaptação o modelo original do BSC para entidades públicas.

Como se observa, diversos estudos nos últimos anos buscaram destacar a importância do BSC na gestão de organizações públicas. Apesar da relevância dos estudos apresentados, pesquisas que avaliam o BSC como ferramenta a ser aplicado na gestão dos municípios, especialmente aqueles de menor porte, são escassas. A presente pesquisa busca contribuir neste processo, por meio do seguinte questionamento: Como a metodologia do BSC, aplicado à gestão de pequenos municípios, pode contribuir para a eficácia na aplicação dos recursos públicos e quais os principais benefícios e obstáculos de sua implantação?

O objetivo geral consiste em apresentar uma estrutura de implantação do BSC e analisar as vantagens e benefícios, bem como, os principais obstáculos na implantação do BSC na gestão dos pequenos municípios. São considerados municípios de pequeno porte aqueles com população até 20 mil habitantes, de acordo com a classificação do Instituto Brasileiro de Geografia e Estatística- IBGE.

A Administração Pública como um todo se ressente de reconhecidos problemas de ineficiência e ineficácia. Apesar de a LRF apresentar um reordenamento das finanças públicas, não apresenta uma visão de longo prazo, especialmente no que diz respeito à conformação estratégica dos órgãos públicos, orientada para elevação consistente de seu desempenho.

Os pequenos municípios brasileiros apresentam um cenário ainda mais desfavorável no que diz respeito à Administração Pública, muitas vezes não conseguindo sequer atender às formalidades legais. Entretanto, na medida em que os recursos reconhecidamente escassos dessas administrações são aplicados com baixos níveis de eficiência e eficácia, as repercussões negativas atingem toda a sociedade. Portanto, a análise e avaliação da aplicação do BSC às administrações dos pequenos municípios podem apresentar oportunas e valiosas contribuições.

Revista Brasileira de Políticas Públicas e Internacionais, v.3, n.2, Dezembro/2018, pp. 59-80. 
TAFFAREL, Marinês. O Balanced Scorecard como Ferramenta Estratégica para Pequenos Municípios.

\section{Revisão de Literatura}

Esta seção apresenta os principais conceitos e discussões voltadas à Administração Pública e ao Balanced Scorecard.

\subsection{Planejamento na Administração Pública Municipal}

Para Andrade (2008) a Administração Pública é todo aparelhamento que busca a realização de serviços que satisfaçam as necessidades coletivas. Os serviços públicos são aqueles que a administração presta diretamente à coletividade de forma direta ou indireta, nas esferas federal, estadual e municipal. Kohama (2006) destaca que a administração pública, baseada em uma estrutura hierarquizada com graduação de autoridade, correspondente às diversas categorias funcionais, ordenada pelo Poder Executivo de forma que distribua e escalone as funções de seus órgãos e agentes, estabelecendo a relação de subordinação.

O planejamento pode ser definido como um processo organizado que expressa metas a serem realizadas dentro de um determinado prazo (Silva, 2008). Para Slomski (2007) o processo de planejamento nos municípios brasileiros ocorre por meio de um conjunto de instrumentos, tais como: o Plano Diretor do Município, o Programa de Governo, o Plano Plurianual, a Lei de Diretrizes Orçamentárias e a Lei de Orçamento Anual. Kohama (2006) destaca que o PPA, a LDO e a LOA, planeja, orienta e executa, respectivamente, as políticas públicas e programas de governo, resultando em serviços públicos necessários para atender a coletividade.

O processo de planejamento na administração pública é composto por um ciclo de ações, que inicia com a elaboração do PPA e termina na efetiva execução orçamentária e financeira. O PPA é o instrumento de planejamento estratégico das ações do governo para um período de quatro anos, sobre o qual são elaborados a LDO que compreende as metas, prioridades e despesas de capital que serão desenvolvidas no exercício financeiro subsequente (Slomski, 2007).

Revista Brasileira de Políticas Públicas e Internacionais, v.3, n.2, Dezembro/2018, pp. 59-80. 
TAFFAREL, Marinês. O Balanced Scorecard como Ferramenta Estratégica para Pequenos Municípios.

A Lei de Diretrizes Orçamentárias é um instrumento de planejamento que estabelece as metas e prioridades da Administração Pública e orienta a elaboração da Lei Orçamentária Anual(LOA.A Lei Orçamentária Anual é de iniciativa do Poder Executivo e estabelece as políticas públicas para o exercício a que se refere. O conteúdo básico desta lei é a estimativa da receita e a fixação da despesa. Assim, a LOA é elaborada a partir da LDO em consonância com o PPA.

\subsection{Avaliação de Desempenho na Administração Pública}

A avaliação de desempenho busca apoiar o processo de tomada de decisão, por meio da introdução de melhorias na prestação de bens e serviços. Na administração pública este processo pode ser estabelecido a partir de indicadores capazes de medir a eficiência, eficácia e efetividade das ações públicas. No Quadro1 são apresentadas algumas diferenças no que se refere à obtenção de resultados na administração pública:

Quadro 1 - Indicadores de resultados na Administração Pública

\section{INDICADORES DE RESULTADOS NA ADMINISTRAÇÃO PÚBLICA}

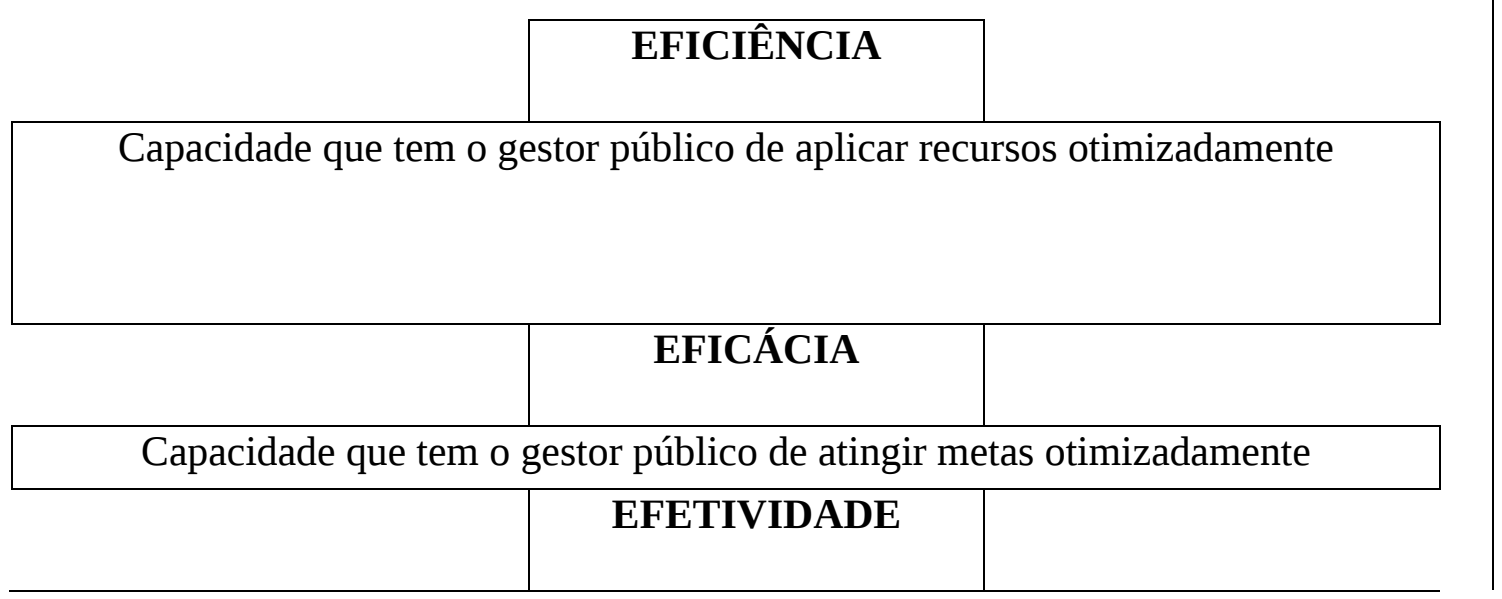

Revista Brasileira de Políticas Públicas e Internacionais, v.3, n.2, Dezembro/2018, pp. 59-80. 
TAFFAREL, Marinês. O Balanced Scorecard como Ferramenta Estratégica para Pequenos Municípios.

Capacidade que tem o gestor público de satisfazer as necessidades da coletividade

Fonte: Bezerra Filho (2006, p. 13)

Os gestores públicos necessitam de um conjunto de informações gerenciais para cumprirem com eficiência, eficácia e efetividade as políticas públicas. Ozório (2003) destaca que instrumentos e metodologias que proporcionem o aperfeiçoamento da gestão estratégica voltada para resultados representam uma demanda urgente na modernização da administração publica.

A busca por maior eficiência nas ações públicas mantém relação estreita com mecanismos de controle, cujo desempenho é medido por meio de indicadores construídos especificamente para a área pública. Para Rocha (2001) torna-se importante avaliar o desempenho da administração pública por meio da construção de indicadores que permitam medir a sua eficiência, eficácia e qualidade, assim como, a criação de sistemas de informação que possibilitem atingir os objetivos e metas planejados. É neste contexto que se destaca o BSC como um instrumento que permite implementar o plano de governo visando atingir os objetivos da administração publica com maior eficiência, eficácia e economicidade.

\subsection{Balanced Scorecad}

Para Kaplan e Norton (1997) o Balanced Scorecard traduz a visão e a estratégia, por meio de um conjunto de medidas de desempenho, de acordo com quatro perspectivas diferentes, as quais equilibram os objetivos da organização de curto e longo prazo, como pode ser observado na Figura 1:

Figura 1: BSC: Tradução da estratégia em termos operacionais

Revista Brasileira de Políticas Públicas e Internacionais, v.3, n.2, Dezembro/2018, pp. 59-80. 
TAFFAREL, Marinês. O Balanced Scorecard como Ferramenta Estratégica para Pequenos Municípios.

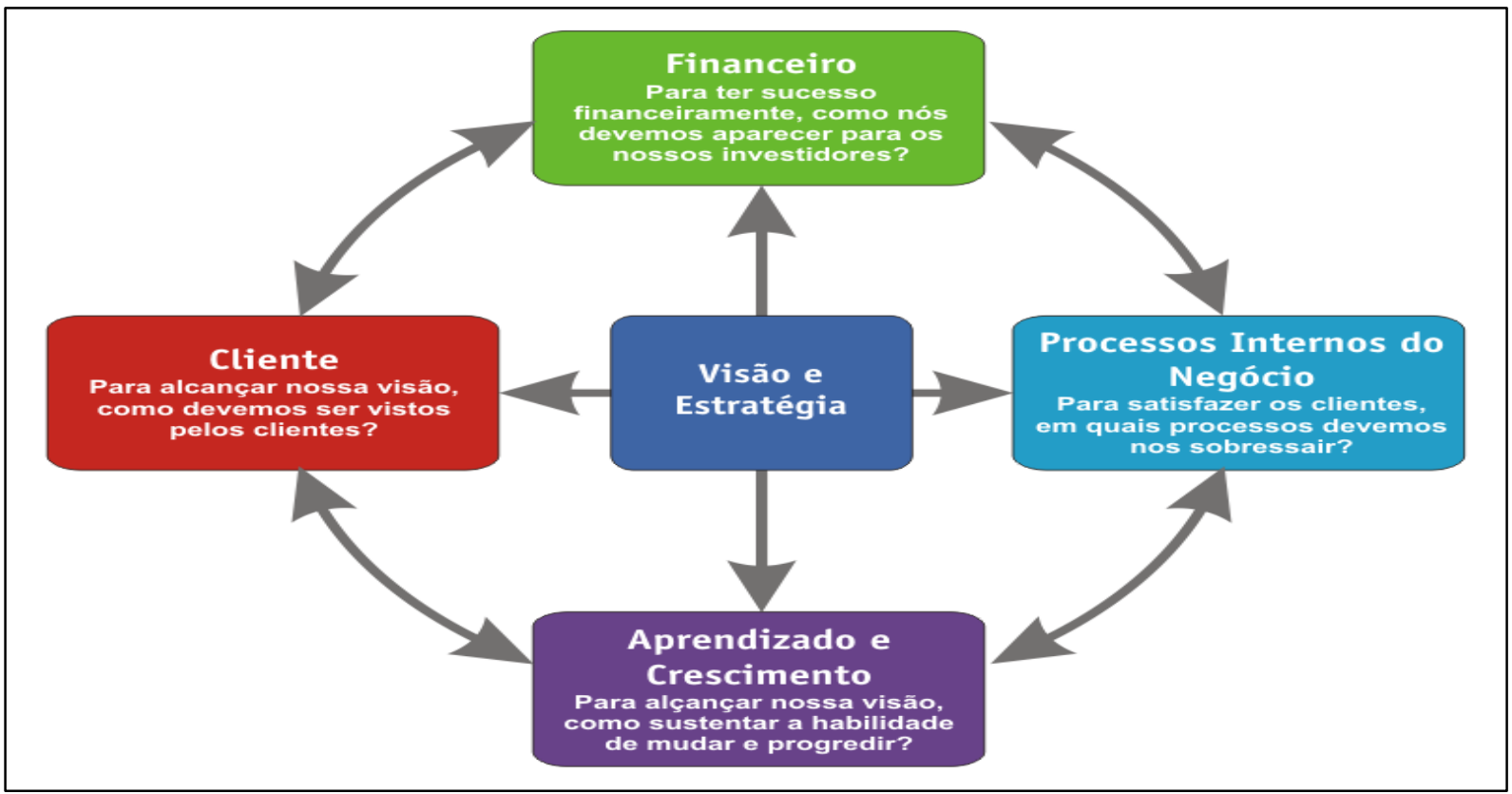

Fonte: Kaplan e Norton (1997)

Kaplan e Norton (1997) referem que o BSC traduz a missão e a estratégia de uma unidade de negócios, conforme mostrado na Figura 2, de acordo com quatro perspectivas:

a) Perspectiva financeira: Indicam se a estratégia está contribuindo para melhoria dos resultados. As medidas financeiras indicam se a estratégia adota pela organização, sua implementação e execução estão contribuído para melhorar os resultados.

b) Perspectiva do cliente: Permite que os executivos identifiquem os segmentos de clientes e mercados nos quais a unidade de negócio competirá. O alcance da perspectiva do cliente é medido pela eficácia e eficiência do atendimento das necessidades da sociedade e não somente pelo desempenho financeiro obtido.

c) Perspectiva dos processos internos: Identificam os processos internos críticos nos quais a empresa deve alcançar a excelência. Neste sentido, visa a identificar os fatores que atraem, mantêm e valorizam os clientes em um segmento-alvo de mercado.

Revista Brasileira de Políticas Públicas e Internacionais, v.3, n.2, Dezembro/2018, pp. 59-80. 
TAFFAREL, Marinês. O Balanced Scorecard como Ferramenta Estratégica para Pequenos Municípios.

d) Perspectiva do aprendizado e crescimento: Identifica a infraestrutura que a empresa deve construir para gerar crescimento e melhoria a longo prazo. Nesta perspectiva, a gestão do conhecimento é importante para disseminar os conhecimentos tácitos e explícitos, buscando utilizar da forma mais eficaz os processos e recursos da Organização.

Para Kaplan e Norton (1997) existe uma relação de causa e efeito que envolvem as perspectivas contidas no modelo, que podem se expressas na seguinte ordem: medidas de aprendizado e crescimento são causadoras de medidas de processos internos, que por sua vez produzem medidas da perspectiva dos clientes, que por sua vez dão origem aos resultados financeiros (Silva \& Abbad, 2011).

A implantação do BSC requer a evidenciação das seguintes medidas gerenciais por meio de um ciclo contínuo, como pode ser observado na Figura 2:

Figura 2: O Balanced Scorecard como estrutura para ação estratégica.

\begin{tabular}{|c|c|c|}
\hline & 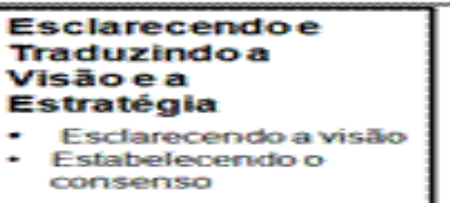 & \\
\hline 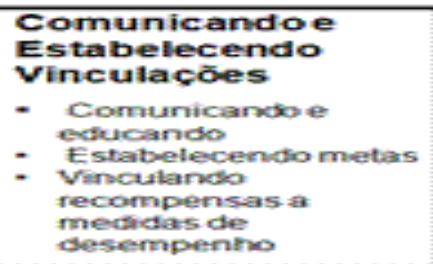 & $\begin{array}{l}\text { Balanced } \\
\text { Scorecard }\end{array}$ & 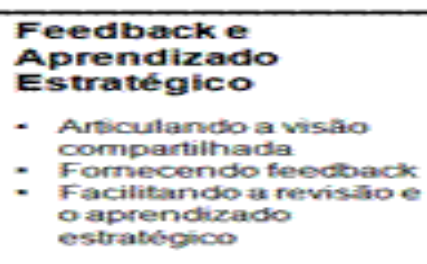 \\
\hline & 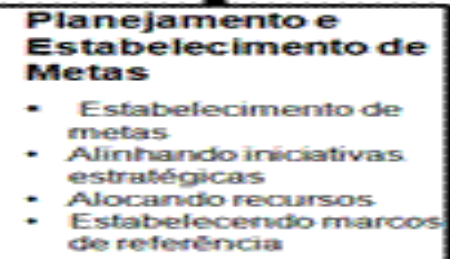 & \\
\hline
\end{tabular}

Fonte: Kaplan \& Norton (1997, p.12).

Revista Brasileira de Políticas Públicas e Internacionais, v.3, n.2, Dezembro/2018, pp. 59-80. 
TAFFAREL, Marinês. O Balanced Scorecard como Ferramenta Estratégica para Pequenos Municípios.

No BSC a estratégia está relacionada com a construção de hipóteses em relação os fenômenos que envolvem causa e efeito nas organizações (Atkinson, 2006). Para Irwin (2002) apesar de terem sido publicados inúmeros casos que refletem o mapeamento de estratégia no setor privado, existe uma lacuna no que se refere à utilização desta técnica no setor público. Assim, embora o foco de resultado financeiro não exista no setor público, é igualmente essencial que todos na organização possuam uma compreensão clara da estratégia e o papel que cada individuo exerce na sua execução.

\subsection{Balanced Scorecard na Administração Pública}

O BSC como instrumento de medição e avaliação do desempenho foi concebido especialmente para o setor privado. No entanto, é possível sua implantação na área pública, a partir de adaptações em sua concepção voltadas a atender as especificações e demandas dessas organizações. Dessa forma, o Balanced Scorecard aplicado na esfera pública requer a inserção de medidas nas dimensões de efetividade, eficácia e eficiência. Haja vista, que na organização pública, a prestação de serviços com excelência envolve a função social (efetividade), qualidade na prestação de serviços (eficácia), e menor consumo de recursos possível (eficiência) (Ghelman \& Reis, 2006).

Para Kaplan e Norton (1997) o BSC pode também proporcionar foco, motivação e responsabilidade em empresas públicas e instituições sem fins lucrativos. Os propositores do modelo enfatizam que, nesse caso, o scorecard oferece a base lógica para a existência dessas organizações (servir clientes e partes interessadas, além de manter os gastos dentro dos limites orçamentários). Além de comunicar externa e internamente os resultados dos vetores de desempenho, pelos quais a organização irá conquistar a sua missão e alcançar os seus objetivos estratégicos.

A distinção entre o setor privado e setor público na implantação do BSC é, que neste último, o cliente é colocado no topo da estrutura. Neste sentido, atingir a missão por meio da satisfação dos clientes/cidadãos é o principal objetivo da gestão. Nesta perspectiva devem ser

Revista Brasileira de Políticas Públicas e Internacionais, v.3, n.2, Dezembro/2018, pp. 59-80. 
TAFFAREL, Marinês. O Balanced Scorecard como Ferramenta Estratégica para Pequenos Municípios.

incluídos objetivos abrangentes da missão organizacional, como redução da pobreza e/ou aumento de empregos (Niven, 2015).

A pesquisa de Santos (2003) buscou apresentar um modelo de BSC voltado para o setor público, o qual inclui a Lei de Responsabilidade Fiscal, de acordo com a Figura 3.

Figura 3 -BSC voltado para o Setor Público

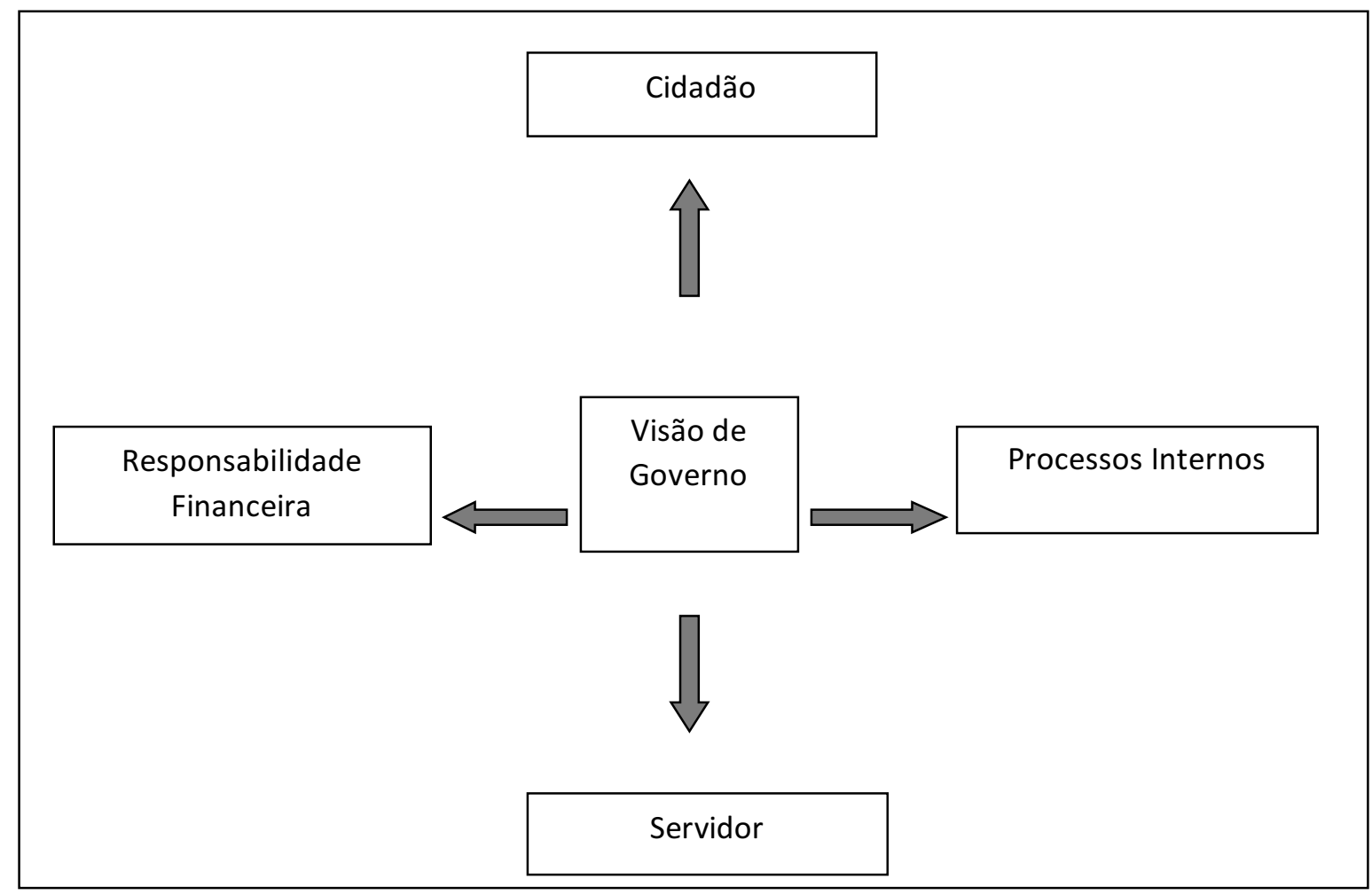

Fonte: Santos (2003)

No modelo, a Perspectiva do Cidadão é voltada para as ações que buscam atender as necessidades coletivas; a Perspectiva da Responsabilidade Financeira está relacionada ao atendimento de projetos e a Lei de Responsabilidade Fiscal; a Perspectiva dos Processos Internos é concernente ao gerenciamento e inovação de processos internos para atender as expectativas

Revista Brasileira de Políticas Públicas e Internacionais, v.3, n.2, Dezembro/2018, pp. 59-80. 
TAFFAREL, Marinês. O Balanced Scorecard como Ferramenta Estratégica para Pequenos Municípios.

do cidadão e da LRF; a Perspectiva do Servidor se refere a sustentação de habilidade e a criatividade dos servidores para atender as expectativas dos cidadãos (Santos, 2003).

Para Ozório (2003) no BSC adaptado à área pública, a primeira perspectiva passa a ser o cliente. Este fato requer, se necessário, a inclusão de dois grupos: o contribuinte e o beneficiário, pois em muitos casos o contribuinte não é o maior beneficiário, ou aquele que apresenta maior dependência dos serviços públicos. Bergue (2005) corrobora este entendimento e propõe a estrutura do BSC para a aplicação em organizações públicas mostrada na Figura 4.

Figura 4: Modelo de BSC para Organizações Públicas

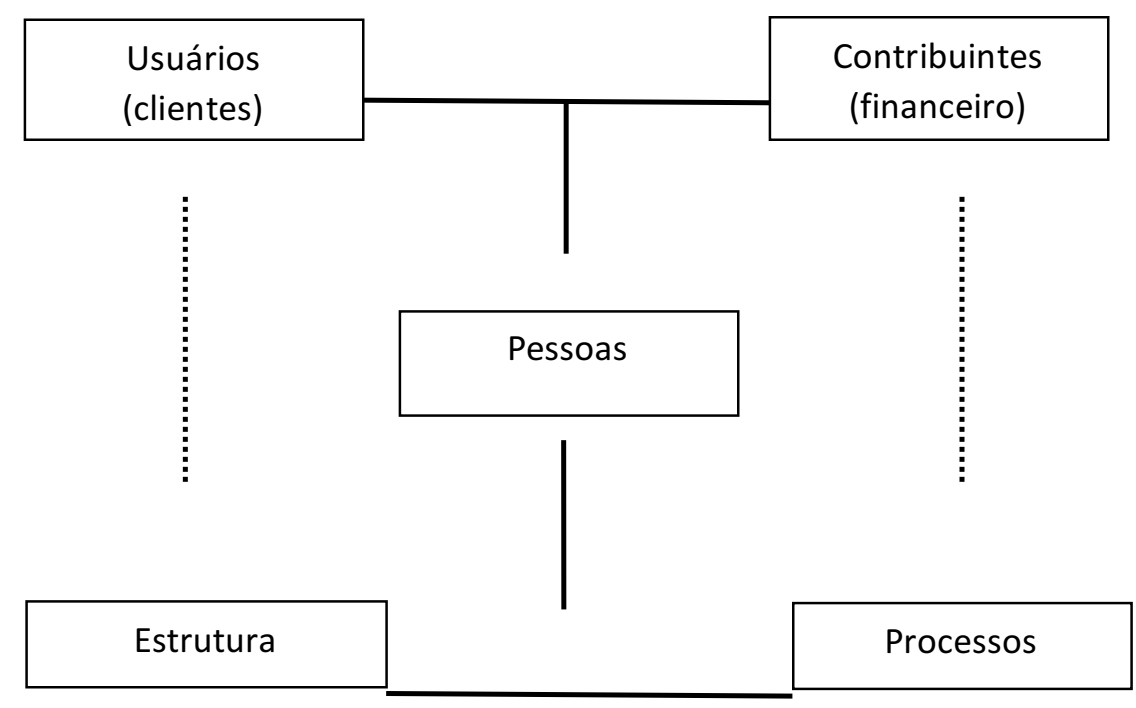

Fonte: Bergue (2005)

Para Bergue (2005) a perspectiva “Pessoas” possui posição de destaque nas dimensões do BSC adaptado para o setor público, situando-se de forma centralizada no modelo. Considerando que a administração pública apresenta estrutura e modelo de gestão orientados por propósitos diferenciados em relação à administração privada, torna-se necessário propor

Revista Brasileira de Políticas Públicas e Internacionais, v.3, n.2, Dezembro/2018, pp. 59-80. 
TAFFAREL, Marinês. O Balanced Scorecard como Ferramenta Estratégica para Pequenos Municípios.

dimensões fundamentais, específicas e relevantes para fins de gerenciamento orientado pela estratégia, como: o usuário/contribuinte; as pessoas; os processos; e a estrutura. Essas dimensões são construídas pelas seguintes considerações: a) o foco do esforço deve estar nos conceitos de planejamento, controle e estratégia; b)observaras diretrizes institucionais existentes(crítica e adaptação) e a interações entre as ações; c) as dimensões gerenciais propostas no BSC tradicional devem ser tomadas como uma orientação básica para a composição de um modelo (também geral) para a administração pública, incorporando os devidos desdobramentos e transformações das dimensões estratégicas originais; e d) cada organização deve transformar o modelo e traduzilo em sistemas corporativos aderentes à sua realidade e necessidades gerenciais.

\section{Procedimentos Metodológicos}

A presente pesquisa tem como objetivo propor um modelo de implantação do BSC para pequenos municípios, preservando as especificidades da área pública. O modelo foi construído a partir do padrão aplicado no setor privado por Kaplan e Norton (1997) e adaptado às particularidades legais e necessidades gerenciais da gestão pública municipal. Trata-se, portanto, de uma pesquisa descritiva, com procedimentos de estudo bibliográfico e documental construído de acordo com a literatura especializada da área, bem como por observação no cotidiano da gestão municipal de um pequeno município.

\section{Modelo Proposto para a Aplicação do BSC em Pequenos Municípios}

Na administração pública municipal, entende-se que a aplicação do BSC deve estar acoplada aos instrumentos legais de planejamento do setor público: Plano Plurianual, Lei de Diretrizes Orçamentárias e Lei Orçamentária Anual ou Orçamento Anual. Assim, além da visão e execução dos planos/estratégia geral de governo, sugere-se que as metas e objetivos sejam descentralizados por área de atuação: Educação, Saúde e Infraestrutura, desmembrado também, os objetivos, indicadores, metas e iniciativas para cada área de ação governamental.

Revista Brasileira de Políticas Públicas e Internacionais, v.3, n.2, Dezembro/2018, pp. 59-80. 
TAFFAREL, Marinês. O Balanced Scorecard como Ferramenta Estratégica para Pequenos Municípios.

Como o principal objetivo da administração pública é a satisfação das necessidades da população, a perspectiva do cidadão/ cliente é colocada como no topo da estrutura do BSC, tal como sugerem Kaplan e Norton (1997) para a área pública. Uma vez aprovados os instrumentos de planejamento, período de um mandato eletivo, o BSC deve ser reconstruído buscando atingir os objetivos do governo municipal.

Na Figura 5, o modelo de aplicação do BSC em pequenos municípios é apresentado.

Figura 5: Estrutura do BSC para pequenos municípios

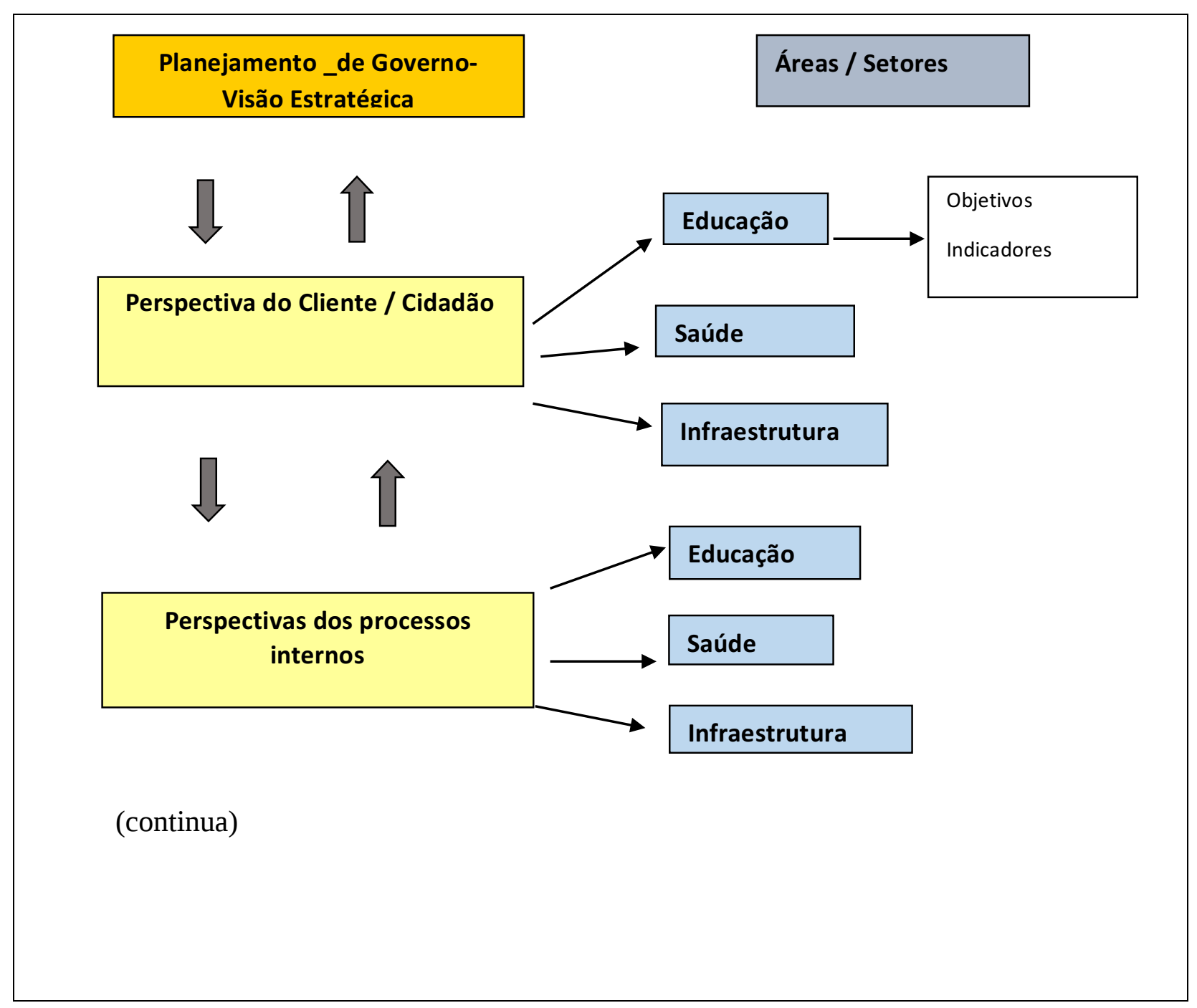

Revista Brasileira de Políticas Públicas e Internacionais, v.3, n.2, Dezembro/2018, pp. 59-80. 
TAFFAREL, Marinês. O Balanced Scorecard como Ferramenta Estratégica para Pequenos Municípios.

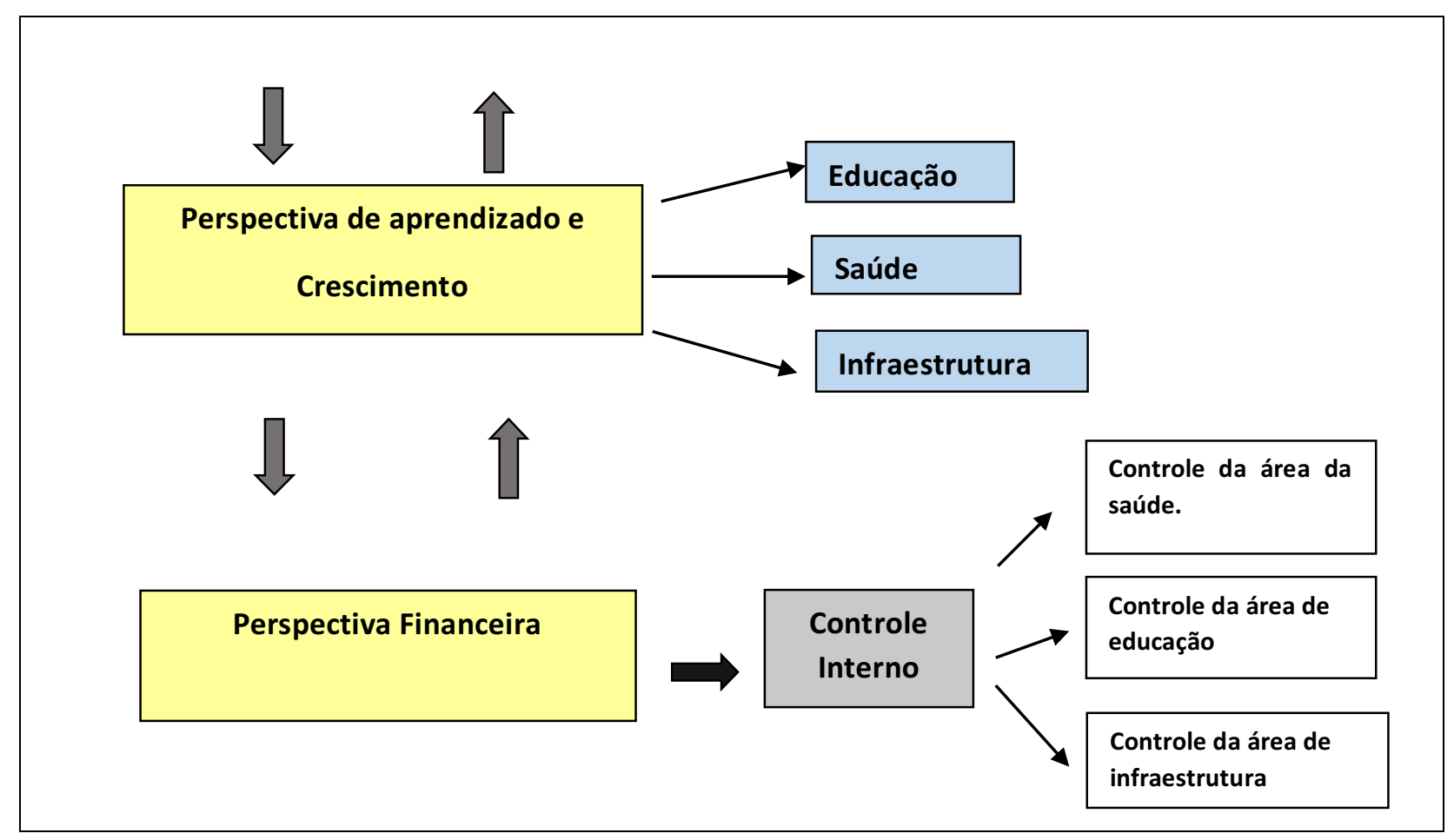

Fonte: A Autora

Considerando que em cada área de atuação as necessidades ou demandas dos cidadãos em relação aos serviços públicos se mostram diferenciadas, torna-se necessário que os planos ou estratégia de governo sejam executados de forma descentralizada. A divisão em áreas de atuação permite melhor acompanhamento dos objetivos, metas e indicadores de governo em diferentes setores, contribuindo para uma maior participação e descentralização de poderes para as secretarias municipais. Neste estudo, são consideradas três grandes áreas para a descentralização: educação, saúde e infraestrutura, no entanto, sugerem-se maiores desdobramentos de acordo com necessidade de gerenciamento.

O BSC aplicado na administração pública de pequenos municípios busca traduzir a visão e os planos ou estratégias do governo municipal, de acordo com as seguintes perspectivas:

Revista Brasileira de Políticas Públicas e Internacionais, v.3, n.2, Dezembro/2018, pp. 59-80. 
TAFFAREL, Marinês. O Balanced Scorecard como Ferramenta Estratégica para Pequenos Municípios.

a) Perspectiva do Cliente / Cidadão: contempla as ações de atendimento das necessidades da população em diferentes áreas, de acordo com a visão e a estratégia de governo. Permite avaliar as demandas dos cidadãos e a satisfação em relação aos serviços prestados.

b) Perspectiva de Processos Internos: voltada à interligação de todas as ações públicas buscando a qualidade dos serviços prestados, além de desenvolver novos mecanismos de controle e redução de custos e proporcionar maior agilidade e qualidade aos serviços públicos.

c) Perspectiva de Aprendizado e Crescimento: busca fortalecer de forma integrada a cooperação e o trabalho em equipe, identificando as potencialidades e a valorização dos profissionais que atuam no setor público.

d) Perspectiva Financeira: interliga as ações da LDO em conformidade com o PPA; efetua a execução do orçamento buscando alavancar as receitas próprias dos municípios para novos investimentos e ações, buscando assegurar a eficiência, efetividade e economicidade dos serviços públicos. O controle interno auxilia nesta função, na medida em que acompanha os limites de gastos com pessoal e endividamento de acordo com a legislação, garantindo que os padrões de qualidade sejam atendidos. No entanto, sugere-se que para uma maior efetividade no acompanhamento financeiro, que esta função seja desdobrada em unidades de controles nas áreas de educação, saúde e infraestrutura, ou de acordo com as necessidades de gerenciamento de cada município.

\subsection{Vantagens e obstáculos da implantação do BSC em pequenos municípios}

A implantação do BSC na administração pública de pequenos municípios apresenta inúmeras vantagens, tendo em vista a necessidade de as decisões e controles das ações públicas serem realizados por meio processos de abrangência gerencial, os quais alinhados a capacidade de tomada e decisão e planejamento dos gestores, permitem que os resultados almejados sejam atingidos. Dentre as vantagens da aplicação do BSC em pequenos municípios destaca-se:

Revista Brasileira de Políticas Públicas e Internacionais, v.3, n.2, Dezembro/2018, pp. 59-80. 
TAFFAREL, Marinês. O Balanced Scorecard como Ferramenta Estratégica para Pequenos Municípios.

a) Traduz o sistema de planejamento integrado do setor público em objetivos e metas, de acordo com as diversas áreas de abrangência e demandas da população local;

b) Maior eficiência e eficácia na aplicação dos recursos públicos, permitindo fomentar melhorias nos serviços públicos e ampliar a sua destinação;

c) Proporciona visão sistêmica do governo municipal e melhorias nos processos que atingem diretamente ou indiretamente os cidadãos;

d) Potencializa melhoria na aplicação dos recursos públicos por meio de um controle de indicadores por área de atuação;

e) Apoio nos processos decisórios e implementação dos planos de governo;

f) Maior efetividade das ações executadas de acordo com a visão e planos de governo;

g) Facilita a comunicação dos objetivos, metas e a avaliação das ações realizadas;

h) Incentiva o crescimento, a profissionalização e o aprendizado contínuo dos servidores públicos.

Para maior efetividade na execução do planejamento público, sugere-se que o BSC aplicado em pequenos municípios seja desdobrado em áreas de atuação, para que as decisões de cada secretaria reflitam os objetivos gerais da gestão municipal.

No entanto, torna-se perceptível, considerando as especificidades da gestão pública, que alguns obstáculos podem interferir no sucesso da implantação do BSC. Possivelmente a escassa ou precária estrutura física, administrativa e financeira e a falta de pessoal especializado sejam as maiores dificuldades, aliadas ao fato de que na administração pública a cada quatro anos existe a possibilidade não só de mudança dos mandatários eleitos, mas também de todo uma situação de governo, o que interfere diretamente na continuidade de estratégias e planos de longo prazo. Além deste fato, o possível despreparo de muitos governantes eleitos e suas equipes de governo, representa um fator limitativo da aplicação do BSC na área publica.

Revista Brasileira de Políticas Públicas e Internacionais, v.3, n.2, Dezembro/2018, pp. 59-80. 
TAFFAREL, Marinês. O Balanced Scorecard como Ferramenta Estratégica para Pequenos Municípios.

\section{Considerações Finais}

As administrações públicas, de maneira geral, carecem de mecanismos de controle e gerenciamento de resultados. Apesar de a LRF ter proporcionado um profundo reordenamento das finanças públicas, orientando para a elevação consistente de seu desempenho, ainda é perceptível a falta de padrões de eficiência e eficácia na execução dos planos de governo.

Para os pequenos municípios brasileiros, este cenário se apresenta ainda mais desfavorável no que diz respeito à administração pública, os quais muitas vezes sequer conseguem atender às exigências legais. Neste sentido, a implantação do Balanced Scorecard como instrumento de tradução da visão, planos e estratégia de governo pode proporcionar inúmeros benefícios, na medida em que contempla as medidas de efetividade, eficiência, eficácia e economicidade dos serviços públicos.

O BSC aplicado para pequenos municípios requer adaptação não apenas para atender às especificidades da área pública, mas também às circunstâncias restritivas que pesam sobre as administrações desses municípios. O modelo de BSC proposto além de trazer a perspectiva do cidadão/cliente como o foco de resultados, requer que os instrumentos de planejamento integrado, PPA, LDO e LOA, sejam acoplados. Para tanto, sugere-se o desdobramento da execução da estratégia para as diferentes áreas de atuação, como: educação, saúde e infraestrutura, proporcionando a descentralização de poderes e responsabilidades ea possibilidade de maior controle dos objetivos e metas de governo.

Em relação aos benefícios da aplicação do BSC em pequenos municípios destaca-se a tradução dos planos e metas de governo de acordo com as diversas áreas de abrangência; maior eficiência e eficácia na destinação dos recursos públicos e o controle das decisões buscando atingir os objetivos propostos. As mudanças da administração pública com a New Public Management estão alinhadas com a cultura de capacitação, descentralização e mensuração de resultados e, sem dúvida estão alinhadas com a adoção do BSC. Entretanto, não se pode esperar que tais mudanças evoluam de forma acelerada por vários motivos, destacando-se, sem dúvida, a cultura predominante que não é favorável e que está profundamente arraigada.

Revista Brasileira de Políticas Públicas e Internacionais, v.3, n.2, Dezembro/2018, pp. 59-80. 
TAFFAREL, Marinês. O Balanced Scorecard como Ferramenta Estratégica para Pequenos Municípios.

Outro fator limitativo importante é a precária continuidade no médio e longo prazo, característica do setor público, devida à substituição dos governos ao final dos mandatos. Isso pode comprometer a implantação do BSC e, de forma mais grave, condenar ao descrédito sua adoção. Ademais, a aplicação do BSC aos municípios de pequeno porte se refere à exiguidade de recursos financeiros e de infraestrutura, precariedade administrativa e financeira, falta de pessoal capacitado e, possível despreparo de administradores públicos eleitos e de suas equipes de governo.

\section{Referências}

Andrade, N. de A. (2008). Contabilidade pública na gestão municipal: Métodos com base na LC $n^{o}$ 101/00 e nas classificações contábeis advindas da SOF e STN. São Paulo: Atlas.

Annapurna, V., \& Manchala, G. (2017). Balanced Scorecard Evaluation of the Performance of Indian Public Sector Banks. Indian Journal of Finance, 11(9), 7-21.

Atkinson, H. (2006). Strategy implementation: a role for the Balanced Scorecard? Management Decision,44 (10), 1441-1460.

Bergue, S. T. (2005). Gestão estratégica de pessoas e Balanced Scorecard em organizações públicas. Revista Análise, 16 (2), 265-284.

Bezerra Filho, J. E. (2006).Contabilidade pública: teoria, técnica de elaboração de balanços e questões. Rio de Janeiro: Elsevier.

Blonski, F., Prates, R. C., \& Costa, M. (2017). Managerial Control Under The Perspective Of New Public Management: The Adoption Of The Balanced Scorecard By The Federal Revenue Service of Brazil (Rfb). Administracão Pública e Gestão Social, 9(1), 15-30.

Bobe, B. J.; Mihret, D. G., \& Obo, D. D. (2017).Public-sector reforms and balanced scorecard adoption: an Ethiopian case study. Accounting, Auditing \& Accountability Journal, 30(6), 12301256.

Constituição da Republica Federativa do Brasil. Recuperado dehttp://www.stf.jus.br/arquivo/cms/legislacaoConstituicao/anexo/CF.pdf

Diel, J. O.,\& Bampi, A. C. (2016). Benchmarking and Combined Balanced Scorecard: Tools for Decision Making in Public Management. Espacios. 37(06),1-15.

Revista Brasileira de Políticas Públicas e Internacionais, v.3, n.2, Dezembro/2018, pp. 59-80. 
TAFFAREL, Marinês. O Balanced Scorecard como Ferramenta Estratégica para Pequenos Municípios.

Dreveton, B. (2013). The advantages of the balanced scorecard in the public sector: beyond performance measurement. Public Money \& Management, 33(2), 131-136.

Gabriel, T. L.,Gheonea, V., \& Mihaela, T. (2017, may). Balanced Scorecard - Strategic Management Tool of Performance in Public Institutions. IMBIMA Conference, Viena, Austria, 29.

Ghelman, S., \&Costa, S. R. R da. (2006, novembro). Adaptando o BSC para o setor público utilizando os conceitos de efetividade, eficácia e eficiência. Anais do Simpósio de Engenharia da Produção, Bauru, SP, Brasil, 12.

Irwin, D. (2002).Strategy mapping in the public sector. Long Range Planning. 35 (6), 637-647.

Kaplan, S. R.; Norton P. D. (1997).A Estratégia em Ação. Balanced Scorecard (23a reimp.).Rio de Janeiro: Editora Campus

Kohama, H. (2006). Contabilidade Pública: teoria e prática (10a ed.). São Paulo: Atlas.

Lei n. 4.320, de 17 de março de 1964. Estatui Normas Gerais de Direito Financeiro para elaboração e controle dos orçamentos e balanços da União, dos Estados, dos Municípios e do Distrito Federal. Recuperado de http://www.planalto.gov.br/ccivil_03/Leis/14320.htm

Lei Complementar n. 101, de 04 de maio de 2000. Estabelece normas de finanças públicas voltadas para a responsabilidade na gestão fiscal e dá outras providências. (Lei de $\begin{array}{llll}\text { Responsabilidade } & \text { Fiscal LRF). } & \text { Recuperado }\end{array}$ dehttp://www.planalto.gov.br/ccivil_03/Leis/lcp/Lcp101.htm

Matias-Pereira, J. (2006).Finanças Públicas: A Política Orçamentária no Brasil(3a ed.). São Paulo: Atlas.

Monteiro, S. S., \& Ribeiro, V. P. L. (2017). The Balanced Scorecard as a tool for environmental management: approaching the business context to the public sector. Management of Environmental Quality: An International Journal, 28 (3), 332-349, doi.org/10.1108/MEQ-112015-0201

Niven, P. R. (2015, 2a ed.). Adapting the balanced scorecard to fit the public and nonprofit sectors. In: Balanced Scorecard Step-by-Step for Government and Nonprofit Agencies. Recuperado de https://onlinelibrary.wiley.com/doi/10.1002/9781119197287.ch2

Ozório, V. L. T. (2003). A Utilização do Balanced Scorecard no Aperfeiçoamento da Administração Pública Gerencial-Estudo de Caso de uma Autarquia Municipal (Dissertação de Mestrado). Universidade Federal do Rio Grande do Sul, Porto Alegre, RS, Brasil.

Revista Brasileira de Políticas Públicas e Internacionais, v.3, n.2, Dezembro/2018, pp. 59-80. 
TAFFAREL, Marinês. O Balanced Scorecard como Ferramenta Estratégica para Pequenos Municípios.

Pinto, R. F. (2008). As Reformas do Estado e o Paradigma da Nova Economia Institucional.In Peixoto, J. P. M. (Org.) Governando o Governo:Modernização da Administração Pública no Brasil. São Paulo: Editora Atlas.

Rocha, J. A. O. (2001).Gestão Pública e Modernização Administrativa. Oeiras. Instituto Nacional de Administração.

Santana, W. F., Pessoa, L. G. S. B., CABRAL, L. M. M. A. C., Santos, S. R. B., \& Diniz, J. A. (2007, setembro). Eficácia orçamentária municipal: os impactos produzidos pela Lei de Responsabilidade Fiscal. Anais do XXXI Encontro da Anpad, Rio de Janeiro, RJ, Brasil.

Santos, N. J. (2003, outubro). Planejamento e orçamento na área pública: uma aplicação doBalanced Scorecard. Anais do XXIII Encontro Nacional de Engenharia de Produção, Ouro Preto, MG, Brasil.

Silva, A. L., \& Abbad, G. S. (2011). Benefícios e limitações do Balanced Scorecard para avaliação de Resultados organizacionais em treinamento, desenvolvimento e Educação. Revista Ibero-Americana de Estratégia - RIAE, São Paulo. 10 (1), 04-28.

Silva, J. S. P. (2008). Planejamento Econômico e Fomento Público: instrumentos para o desenvolvimento nacional(Dissertação de Mestrado). Universidade Federal do Paraná, Curitiba, PR, Brasil.

Slomski, V. (2007). Controladoria e Governança na Gestão Pública (1a ed.). São Paulo: Atlas.

Wen-Cheng, L. (2013). Balanced Scorecard and IPA Enables Public Service in Township Management: Local Government Performance. Lex Localis. Journal of Local SelfGovernment.11 (1), 21-32.

Revista Brasileira de Políticas Públicas e Internacionais, v.3, n.2, Dezembro/2018, pp. 59-80. 